\title{
Optical Fock-state synthesizer
}

\author{
G. M. D'Ariano, L. Maccone, M. G. A. Paris, and M. F. Sacchi \\ Theoretical Quantum Optics Group, INFM and Dipartimento di Fisica "A. Volta" \\ Università di Pavia \\ Via A. Bassi 6, I-27100 Pavia, Italy
}

(November 20, 2018)

\begin{abstract}
We suggest a tunable optical device to synthesize Fock states and their superpositions starting from a coherent source. The scheme involves an avalanche triggering photodetector and a ring cavity coupled to a traveling wave through a cross-Kerr medium. Low quantum efficiency at the photodetector improves the synthesizing quality at the expense of reducing the synthesizing rate.
\end{abstract}

\section{INTRODUCTION}

In the last decade, much attention has been devoted to the quantum engineering of nonclassical light. Different types of nonclassical states can be now prepared, and their quantum properties can be entirely characterized by accessible measurement schemes. Among the various quantum states of the optical field, states with a definite number of photons deserve a special attention. Indeed, besides the interest for fundamental tests of Quantum Mechanics [1], the Fock (number) states are also relevant for many applications, as for example, to achieve the optimal capacity coding in quantum communication channels [2], or the Heisenberg sensitivity limit in high-precision quantum interferometry [3].

Different methods for the generation of Fock states have been proposed, both for traveling-wave and cavity fields. For traveling-wave fields, these methods are principally based on tailored nonlinear interactions [1], conditional measurements [5], or state engineering [6]. The experimental realizability or effectiveness of these proposals is often challenging. On the other hand, Fock states have been generated into cavities [7], i.e. using the micromaser trapped states.

In this paper we address the problem of Fock state generation in a traveling mode. We suggest an optical device based on an avalanche triggering photodetector and a ring cavity coupled to an external traveling wave through a cross-Kerr medium. Remarkably, the input states of our proposal are just customary coherent states. Our scheme differs from conventional setups involving conditional photon counters [5], since we use simple on-off detection of an intense coherent field, allowing very low quantum efficiency at the photodetector. Moreover, as we will see, the scheme can also be used to engineer superpositions of few Fock states, which are a crucial resource for optical quantum computers [8], and quantum tomography of optical Hamiltonians [9].

The main advantages of the present scheme are its tunability in preparing any chosen number state and selected superpositions, along with its robustness against the imperfections of the triggering photodetector. Indeed, low quantum efficiency at the photodetector has no detrimental effect on the filtering process, but only reduces the state-synthesizing rate.

The paper is structured as follows. In the next Section the scheme is introduced, and its use in preparing Fock states is described. In Section III we show how the same scheme can be used to prepare superpositions of few Fock states, whereas in Section $\mathbb{I V}$ the effects of non-unit quantum efficiency at the photodetector are taken into account. Section $\square$ closes the paper by summarizing results, and discussing the feasibility of the scheme.

\section{SYNTHESIS OF FOCK STATES}

The schematic setup of the synthesizer is depicted in Fig. 1. The ring cavity is build by two mirrors and two high reflectivity beam splitters. Here, for simplicity, we suppose that the beam splitters have the same transmissivity $\tau$. The cavity is fed by a coherent state in the mode $a_{1}$, whereas the mode $a_{2}$ is left unexcited. Through the cross-Kerr interaction, the cavity mode $d$ is coupled to an external traveling mode $c_{1}$, according to the unitary evolution [10]

$$
\hat{U}_{K}=\exp \left(-i \chi t d^{\dagger} d c_{1}^{\dagger} c_{1}\right)
$$

$\chi$ being the nonlinear susceptibility of the medium and $t$ the interaction time. The signal mode $c_{1}$ is prepared in a coherent state, and, additionally, a tunable phase shift $\psi$ is introduced in the cavity mode. 
The scheme is suitable for applications with both continuous waves and pulses. In a situation involving pulses, however, in order have states with a definite number of photons we need a long enough quantization time. Indeed, the coherence time of the signal mode should be quite long compared to the probe one. We assume that the coherence time of the input signal is of the order of the photon flight time in the cavity, thus assuring that the cavity mode effectively couples with the signal through the cross-Kerr medium. Moreover, the coherence time of the cavity mode should be shorter than the signal one, thus allowing the Kerr phase to cumulate in the loop. In summary, the coherence time of the probe mode should be shorter than the signal one, which, in turn, is determined by the photon flight time in the cavity.

At the output of the cavity the field is monitored by an avalanche photodetector. For the purpose of our scheme, we only need to verify the presence or absence of the field, at the output port of the cavity through the triggering photodiode D. Let us initially assume unit quantum efficiency at photodetection. The measurement is described by the two-value probability operator measure (POM) $\hat{\Pi}_{n}$

$$
\hat{\Pi}_{0} \doteq|0\rangle\left\langle 0\left|, \quad \hat{\Pi}_{1} \doteq \hat{I}_{b_{2}}-\right| 0\right\rangle\langle 0|,
$$

where $|0\rangle$ is the vacuum and $\hat{I}_{b_{2}}$ is the identity for mode $b_{2}$. As we will show in the following, due to the very steep dependence of the cavity transmissivity on the total phase shift-including both cross Kerr interaction and phase $\psi$ - the detection of the field at photodetector D guarantees that the free mode $c_{2}$ at the output of the Kerr medium is reduced into a Fock state or a superposition of few Fock states.

The mode transformations of the ring cavity are [11]

$$
\left\{\begin{array}{l}
b_{1}=\kappa(\varphi) a_{1}+e^{i \varphi} \sigma(\varphi) a_{2} \\
b_{2}=\sigma(\varphi) a_{1}+\kappa(\varphi) a_{2}
\end{array}\right.
$$

where the phase-dependent cavity transmissivity $\sigma$ and reflectivity $\kappa$ are given by

$$
\begin{aligned}
& \kappa(\varphi) \doteq \frac{\sqrt{1-\tau}\left(e^{i \varphi}-1\right)}{1-e^{i \varphi}(1-\tau)} \\
& \sigma(\varphi) \doteq \frac{\tau}{1-e^{i \varphi}(1-\tau)}
\end{aligned}
$$

with $|\kappa(\varphi)|^{2}+|\sigma(\varphi)|^{2}=1$. The transformations (3) and (4) are rigorously obtained by quantizing the e.m. field modes which solve the Helmholtz equation of the etalon, as in Ref. [1] , and taking the input/output modes of the asymptotic free plane waves. However, a naive solution of the etalon as a loop of beam splitters gives the same result, with the internal modes having a reduced commutator (this point is well explained in Ref. [12]). For $c_{1}$ in the Fock state $|n\rangle$, the total phase shift is given by

$$
\varphi=\psi-\chi n t \equiv \varphi_{n}
$$

To simplify the notation, we write $\sigma_{n} \doteq \sigma\left(\varphi_{n}\right)$ and analogously for $\kappa$. Let us now consider the input state

$$
\hat{\varrho}_{i n}=|\alpha\rangle\langle\alpha|\otimes| 0\rangle\langle 0| \otimes \hat{\nu}_{i n},
$$

namely a generic state $\hat{\nu}_{i n}$ for mode $c_{1}$, a coherent state $|\alpha\rangle$ for mode $a_{1}$, and vacuum for $a_{2}$. In the Schrödinger picture the output state can be written in the form

$$
\hat{\varrho}_{\text {out }}=\sum_{n, m=0}^{\infty} \nu_{n m}\left|\kappa_{n} \alpha\right\rangle\left\langle\kappa_{m} \alpha|\otimes| \sigma_{n} \alpha\right\rangle\left\langle\sigma_{m} \alpha|\otimes| n\right\rangle\langle m| .
$$

The process of filtering the desired Fock state from the input state $\hat{\nu}_{i n}$ is triggered by the photodetector D as follows. The probabilities corresponding to the outcomes 1 (detector D on) and 0 (detector D off) are given by

$$
P_{1}=\operatorname{Tr}\left[\hat{\varrho}_{\text {out }} \hat{\Pi}_{1}\right]=\sum_{n=0}^{\infty} \nu_{n n}\left(1-e^{-|\alpha|^{2}\left|\sigma_{n}\right|^{2}}\right),
$$

and $P_{0}=1-P_{1}$. By means of Eq. (4) we have

$$
\left|\sigma_{n}\right|^{2}=\left(1+4 \frac{1-\tau}{\tau^{2}} \sin ^{2} \frac{\psi-\chi n t}{2}\right)^{-1}
$$


which is a periodic function sharply peaked at

$$
n=\frac{\psi+2 \pi j}{\chi t} \doteq n^{*}+\frac{2 \pi}{\chi t} j, \quad j \in \mathbb{Z},
$$

with unit maximum height and width of the same order of the beam splitter transmissivity $\tau$ (typically $\tau \sim 10^{-4} \div$ $10^{-6}$ ). The value $n^{*}$ in Eq. (10) can be adjusted to an arbitrary integer by tuning the phase-shift $\psi$ as a multiple of $\chi t$, whereas multiple resonances are avoided by using small nonlinearity $\chi t \ll 1$, so that the values of $n$ satisfying Eq. (10) for $j \neq 0$ correspond to vanishing matrix elements $\nu_{n i} \simeq 0 \forall i$. In this case for high-quality cavities $\tau \ll \chi t$ in Eq. (8) we have $\left|\sigma_{n}\right|^{2} \simeq \delta_{n n^{*}}$, and the detection probability $P_{1}$ rewrites $P_{1} \simeq \nu_{n^{*} n^{*}}\left(1-e^{-|\alpha|^{2}}\right)$. Notice that increasing the amplitude of $\alpha$ will enhance the detection probability $P_{1}$. Moreover, one can optimize also the input state $\hat{\nu}_{\text {in }}$ to achieve the highest $\nu_{n^{*} n^{*}}$. For example, in the case of a coherent input $\hat{\nu}_{i n}=|\beta\rangle\langle\beta|$, one could select $|\beta| \simeq \sqrt{n^{*}}$.

We now evaluate the conditional state $\hat{\nu}_{\text {out }}$ at the output of the Kerr medium for detector D on. One has

$$
\hat{\nu}_{\text {out }}=\frac{1}{P_{1}} \operatorname{Tr}_{a_{1} a_{2}}\left[\hat{\varrho}_{\text {out }} \hat{\Pi}_{1}\right]=\frac{e^{-|\alpha|^{2}}}{P_{1}} \sum_{n, m=0}^{\infty} \nu_{n m} e^{|\alpha|^{2} \kappa_{n} \kappa_{m}^{*}}\left(e^{|\alpha|^{2} \sigma_{n} \sigma_{m}^{*}}-1\right)|n\rangle\langle m|,
$$

where the partial trace is performed over the ring cavity modes. The argument $\theta$ of $\sigma(\varphi)=|\sigma(\varphi)| \exp [i \theta(\varphi)]$ is given by $\theta(\varphi)=\arctan \left[\frac{(1-\tau) \sin \varphi}{1-(1-\tau) \cos \varphi}\right]$. For $\tau \ll 1$, as already noticed, $\left|\sigma_{n}\right|$ is nonzero only for $n=n^{*}$, and correspondingly we have $\theta\left(\varphi_{n}\right)=0$. Therefore, for all practical purposes we can write $\sigma_{n} \sigma_{m}^{*} \simeq\left|\sigma_{n}\right|\left|\sigma_{m}^{*}\right| \simeq \delta_{n n^{*}} \delta_{m n^{*}}$, and the output state (11) becomes

$$
\hat{\nu}_{\text {out }} \simeq\left|n^{*}\right\rangle\left\langle n^{*}\right|, \quad \tau \ll \chi t
$$

i.e . the Fock component $\left|n^{*}\right\rangle$ has been filtered from the initial state $\hat{\nu}_{i n}$.

In Fig. 2 we report the number distribution of the conditional output state $\hat{\nu}_{\text {out }}$, with $\psi$ tuned to obtain $\left|n^{*}=4\right\rangle$ for different values of the beam splitter transmissivity.

\section{SYNTHESIS OF SUPERPOSITIONS}

We now show how the same setup may be used to produce superpositions of Fock states. By choosing higher nonlinearities, the quantity $2 \pi /(\chi t)$ decreases and $\left|\sigma_{n}\right|^{2}$ can be significantly different from zero for more than one value of $n$ corresponding to sizeable components of the input state $\hat{\nu}_{i n}$. If there are only two of these "resonant" values $n_{1}=n^{*}$ and $n_{2}=n^{*}+2 \pi / \chi t$, we have $\left|\sigma_{n}\right| \simeq \delta_{n n_{1}}+\delta_{n n_{2}}$ and $P_{1}$ now reads

$$
P_{1} \simeq\left(\nu_{n_{1} n_{1}}+\nu_{n_{2} n_{2}}\right)\left(1-e^{-|\alpha|^{2}}\right), \quad \tau \ll \chi t .
$$

Accordingly, the conditional state after a successful photodetection becomes

$$
\begin{aligned}
& \hat{\nu}_{\text {out }} \simeq \frac{1}{\nu_{n_{1} n_{1}}+\nu_{n_{2} n_{2}}}\left[\nu_{n_{1} n_{1}}\left|n_{1}\right\rangle\left\langle n_{1}\left|+\nu_{n_{2} n_{2}}\right| n_{2}\right\rangle\left\langle n_{2}\right|\right. \\
& \left.+\nu_{n_{1} n_{2}}\left|n_{1}\right\rangle\left\langle n_{2}\left|+\nu_{n_{2} n_{1}}\right| n_{2}\right\rangle\left\langle n_{1}\right|\right] \quad \tau \ll \chi t,
\end{aligned}
$$

which is a pure state if and only if $\nu_{n_{1} n_{1}} \nu_{n_{2} n_{2}}=\nu_{n_{1} n_{2}} \nu_{n_{2} n_{1}}$, namely for $\hat{\nu}_{i n}$ in a pure state. In Fig. 3 we report the density matrix of the conditional output state $\hat{\nu}_{\text {out }}$, with $\psi$ tuned to obtain a superposition of the Fock states $\left|n_{1} \equiv 10\right\rangle$ and $\left|n_{2} \equiv 20\right\rangle$ for different values of the beam splitter transmissivity. It is worth noting that the coefficients of the superposition in Eq. (14) are selected by the input state $\hat{\nu}_{i n}$. Therefore, in order to have a superposition with equal weights starting from a coherent state $|\beta\rangle$, it is sufficient to choose its amplitude in such a way that $|\beta|^{2}=\left(n_{1} ! / n_{2} !\right)^{1 /\left(n_{1}-n_{2}\right)}$. Notice, however, that with this choice of $\beta$ we find a small contribution due to the term with $j=2$ in Eq. (10). 


\section{EFFECTS OF IMPERFECT PHOTODETECTION}

Let us now take into account the quantum efficiency $\eta$ at the photodetector. In this case the POM $\hat{\Pi}_{n}$ is replaced with $\hat{\Pi}_{n}^{(\eta)}$, where

$$
\hat{\Pi}_{0}^{(\eta)} \doteq \sum_{k=0}^{\infty}(1-\eta)^{k}|k\rangle\langle k|, \quad \hat{\Pi}_{1}^{(\eta)} \doteq I_{b_{2}}-\hat{\Pi}_{0}^{(\eta)} .
$$

The probability $P_{1}^{(\eta)}$ of having outcome 1 at the photodetector and the conditional output state now are the following

$$
\begin{aligned}
& P_{1}^{(\eta)}=\operatorname{Tr}\left[\hat{\Pi}_{1}^{(\eta)} \hat{\varrho}_{\text {out }}\right]=\sum_{n=0}^{\infty} \nu_{n n}\left(1-e^{-\eta|\alpha|^{2}\left|\sigma_{n}\right|^{2}}\right), \\
& \hat{\nu}_{\text {out }}^{(\eta)}=\frac{e^{-|\alpha|^{2}}}{P_{1}^{(\eta)}} \sum_{n, m=0}^{\infty} \nu_{n m} e^{|\alpha|^{2}\left[\kappa_{n} \kappa_{m}^{*}+\sigma_{n} \sigma_{m}^{*}\right]}\left(1-e^{-\eta|\alpha|^{2} \sigma_{n} \sigma_{m}^{*}}\right)|n\rangle\langle m| .
\end{aligned}
$$

Remarkably, low values for the quantum efficiency $\eta$ can reinforce the process of filtering, though at the cost of lowering the probability $P_{1}^{(\eta)}$ of photodetection. In fact, $\eta$ scales the term $\sigma_{p} \sigma_{q}^{*}$ in the exponential in Eq. (17), thus lowering the off-resonance contributions. In Fig. 目a we actually purify the superposition shown in Fig. 3a by lowering the quantum efficiency to the value $\eta=20 \%$. The probability of obtaining the state is correspondingly lowered from the value $P_{1}=0.205$ to $P_{1}^{(\eta)}=0.116$. An analogous argument holds for the dependence of the output state $\hat{\rho}_{\text {out }}$ on

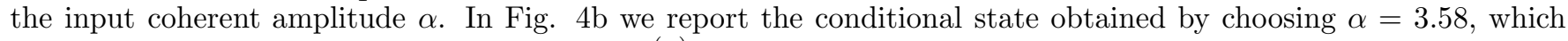
corresponds to the same detection probability $P_{1}^{(\alpha)}=0.116$. Obviously the above discussion on the effect of non-unit quantum efficiency and on the intensity of the input coherent state $|\alpha\rangle$ holds also for the generation of single Fock states.

\section{CONCLUSIONS AND OUTLOOK}

The present proposal is characterized by two relevant features: its tunability in the preparation of any chosen number state and of selected superpositions, and its robustness against the imperfections of the photodetection process. Therefore, it is a matter of interest to discuss its feasibility in practical applications. This mostly depends on two parameters: the quality factor of the ring cavity (governed by the beam splitters transmissivity $\tau$ ) and the value of the nonlinear coupling $\chi t$. For a good cavity (i.e. a cavity with large quality factor) $\tau$ should be quite small, usual values achievable in quantum optical labs being of the order of $\tau \simeq 10^{-4}-10^{-6}$. Remarkably, losses due to absorption processes are of the order of $10^{-7}$, at least one order of magnitude smaller than $\tau$. The scheme also requires an appreciable value for the nonlinear cross-Kerr coupling between the signal and the cavity modes. The coupling can be realized by optical nonlinear couplers, either codirectional or contradirectional, in the case of an all-optical fiber implementation, or by direct matching of modes on a third-order crystal in the case of a free field. Usually, third-order nonlinearities are small, and may be masked by the concurrent self-modulation and absorption processes. Although the effects of self phase modulation can be avoided using resonant $\chi^{(3)}$ media $\sqrt{10}$, currently available nonlinearities can be used in the present scheme only for the preparation of number states [13]. However, recent breakthroughs in nonlinear Kerr physics [14.15] open new perspectives for applications in quantum optics. In fact, methods based on dark atomic resonance and electromagnetically induced transparency have been suggested to strongly enhance nonlinearity while suppressing absorption. These results (especially for atomic gases) indicate that giant Kerr nonlinear shifts of the order of 1 radiant per photon may be obtained by methods not too far from present technology [15, 16 .

As regards the power of the output signal (and the probe mode), we mention that this should not be too large, since the $2 \pi$-periodic resonance structure of the cavity transmissivity relies on the linear phase-shift imposed to the cavity mode. If the power is too large the nonlinear contribution drastically alters this situation, and gives rise to bistability or period-doubling instabilities. The actual value of the allowed power strongly depends on the specific nonlinear crystal employed in the setup. We do not discuss here the technical details.

In conclusion, we have suggested a novel scheme to generate arbitrary optical photon-number eigenstates in a traveling wave mode. The scheme uses on-off photodetection of the field mode exiting a high-Q cavity, which, in turn, is coupled to the traveling-wave by nonlinear Kerr interaction. The input fields for the setup are just customary coherent states. After a successful photodetection, the traveling mode is found in a photon-number eigenstate, or, 
for sufficiently high Kerr nonlinearity, in a superposition of Fock states. The photodetector is needed just to test the presence of an intense coherent output field and, in fact, we have shown that non-unit quantum efficiency at photodetection improves the quality of the state synthesis, however at the expenses of the synthesizing rate.

[1] Quantum Interferometry, F. De Martini et al, Eds. (VCH, Wenheim 1996).

[2] See, for example, C. M. Caves and P. D. Drummond, Rev. Mod. Phys. 66, 481 (1994), and references therein.

[3] M. J. Holland, K. Burnett, Phys. Rev. Lett. 71, 1355 (1993).

[4] S. Y. Kilin and D. B. Horosko, Phys. Rev. Lett. 74, 5206 (1995); W. Leonski, S. Dyrting, and R. Tanas, J. Mod. Opt. 44, 2105 (1997); A. Vidiella-Barranco, and J. A. Roversi, Phys. Rev. A 58 , 3349 (1998); W. Leonski, Phys. Rev. A 54, 3369 (1969).

[5] M. G. A. Paris, Int. J. Mod. Phys. B 11, 1913 (1997); M. Dakna, T. Anhut, T. Opatrny, L. Knoll, and D. G. Welsch, Phys. Rev. A 55, 3184 (1997).

[6] K. Vogel, V. M. Akulin, and W. P. Scheich, Phys. Rev. Lett. 71, 1816 (1993).

[7] J. Krause, M. O. Scully, T. Walther, and H. Walther, Phys. Rev. A 39, 1915 (1989); M. Kozierowski, and S. M. Chumakov, Phys. Rev. A 52, 4194 (1995); P. Domokos, M. Brune, J. M. Raimond, and S. Haroche, Eur. Phys. J. D 1, 1 (1998).

[8] C. H. Bennett and B. P. Di Vincenzo, Nature 377, 389 (1995).

[9] G. M. D'Ariano and L. Maccone, Phys. Rev. Lett. 80, 5465 (1998).

[10] N. Imoto, H. A. Haus, and Y. Yamamoto, Phys. Rev. A 32, 2287 (1985).

[11] M. Ley and R. Loudon, J. Mod. Opt. 34227 (1987).

[12] S. M. Barnett, C. R. Gilson, B. Huttner, and N. Imoto, Phys. Rev. Lett. 77, 1739 (1997).

[13] As an order of magnitude for the parameters involved, consider that an electric field $E \sim 10^{2} \mathrm{~V} / \mathrm{m}$ is achievable for a meter long $\sim 10$ photon pulse with a beam cross section of $\sim 10^{-10} \mathrm{~m}^{2}$, corrisponding to a pulse power per unit area of 1 $\mathrm{mW} / \mathrm{cm}^{2}$ and a frequency $\omega \sim 10^{15} \mathrm{~Hz}$ (see 15 ).

[14] Q. A. Turchette, C. J. Hood, W. Lange, H. Mabuchi, and H. J. Kimble, Phys. Rev. Lett. 75, 4710 (1995); H. Schmidt and A. Imamoglu, Opt. Lett. 21, 1936 (1996)

[15] L. V. Hau, S. E. Harris, Z. Dutton, and C. H. Behroozi, Nature 397, 594 (1999).

[16] A. Imamoglu, H. Schmidt, G. Woods and M. Deutsch, Phys. Rev. Lett. 791467 (1997).

FIG. 1. Schematic diagram of the setup for the generation of Fock states and superposition of Fock states. BS $\mathrm{BS}_{1}$ and $\mathrm{BS}_{2}$ denote high transmissivity beam splitters, and $\psi$ a tunable phase shift. The cavity input modes $a_{1}$ and $a_{2}$ are in a coherent state and in the vacuum respectively. The box denotes the cross Kerr medium that couples the cavity mode $d$ with the traveling free mode $c_{1}$ which is initially prepared in a coherent state. After a successful detection at the photodiode D, which tests the presence of an output excited coherent field in mode $b_{2}$, the output mode $c_{2}$ is found in a Fock state or in a superposition of few Fock states.

FIG. 2. The number distribution of the conditional output state $\hat{\nu}_{\text {out }}$ for different values of the BS transmissivity $\tau$, indicated on each plot. The parameters are chosen to select the number state $\left|n^{*} \equiv 4\right\rangle$, with $\chi t=0.01$ and $\psi=0.04$. The modes $a_{1}$ and $c_{1}$ are both excited in a coherent state with real amplitude $\alpha=20$ and $\beta=2$, respectively. The probabilities of obtaining the three states (i.e. the detection probability at D) are respectively $P_{1}=0.99885,0.4905,0.1997$.

FIG. 3. The density matrix of the conditional output state $\hat{\nu}_{\text {out }}$ for different values of the BS transmissivity. The parameters are chosen to select the superposition $2^{-1 / 2}(|10\rangle+|20\rangle)$, that is $\chi t=\pi / 5$ and $\psi=0$. The modes $a_{1}$ and $c_{1}$ are both excited in a coherent state with amplitude $\alpha=8.0$ and $\beta=(20 ! / 10 !)^{1 / 20} \simeq 3.902$, respectively. The detector has unit quantum efficiency. Notice the small components due to the Fock state $|30\rangle$. The probabilities of synthesizing these two states are $P_{1}=0.205$ and 0.092 , respectively.

FIG. 4. The density matrix of the conditional output state $\hat{\nu}_{\text {out }}$ as in Fig. $3 a$. Here we show the matrix elements for lowered quantum efficiency $\eta=20 \%$ in (a), and for lowered input amplitude $\alpha=3.58$ in (b). Notice, in both cases, the improvement over the reconstructed state of Fig. 3 a. 


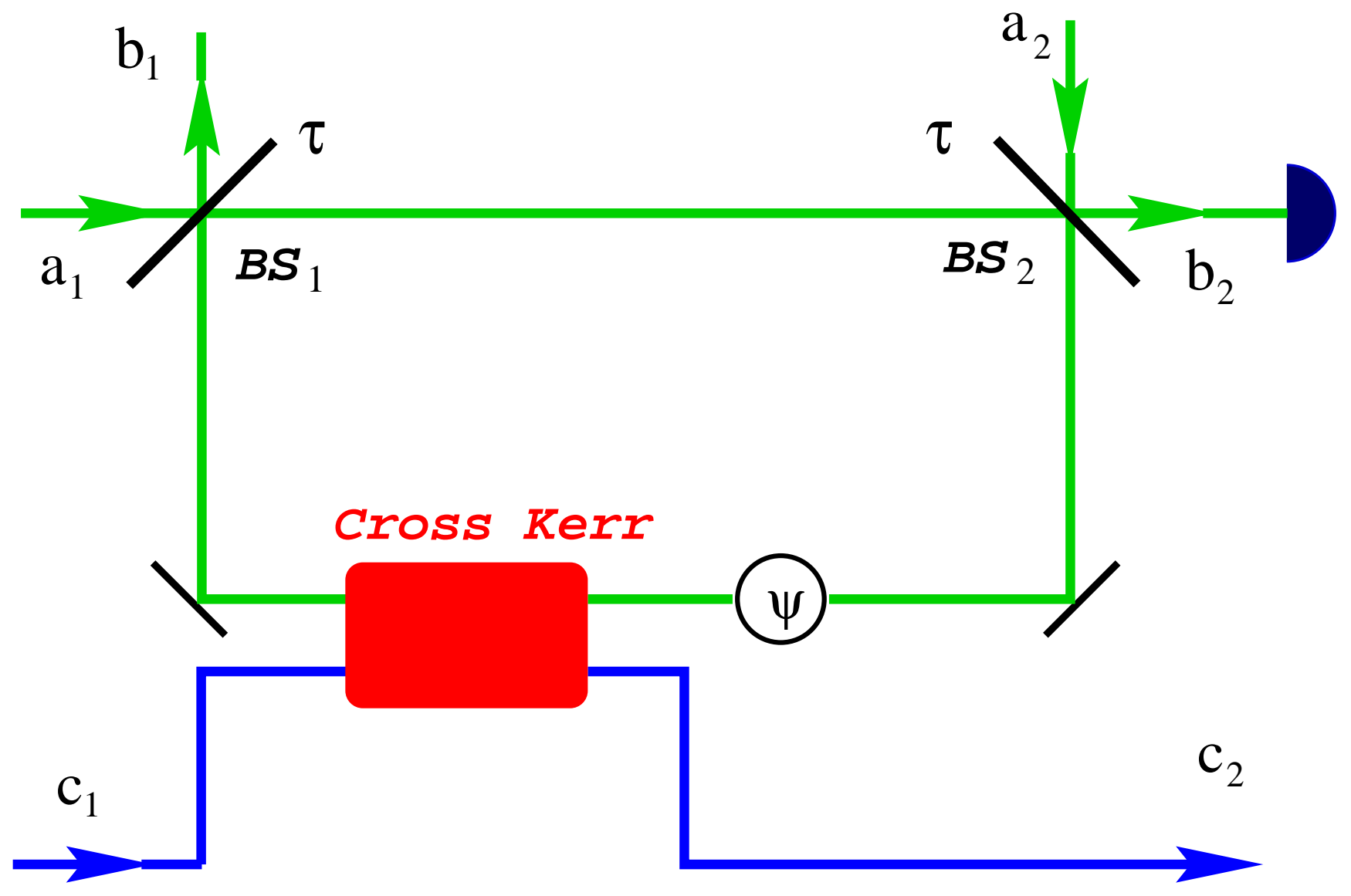




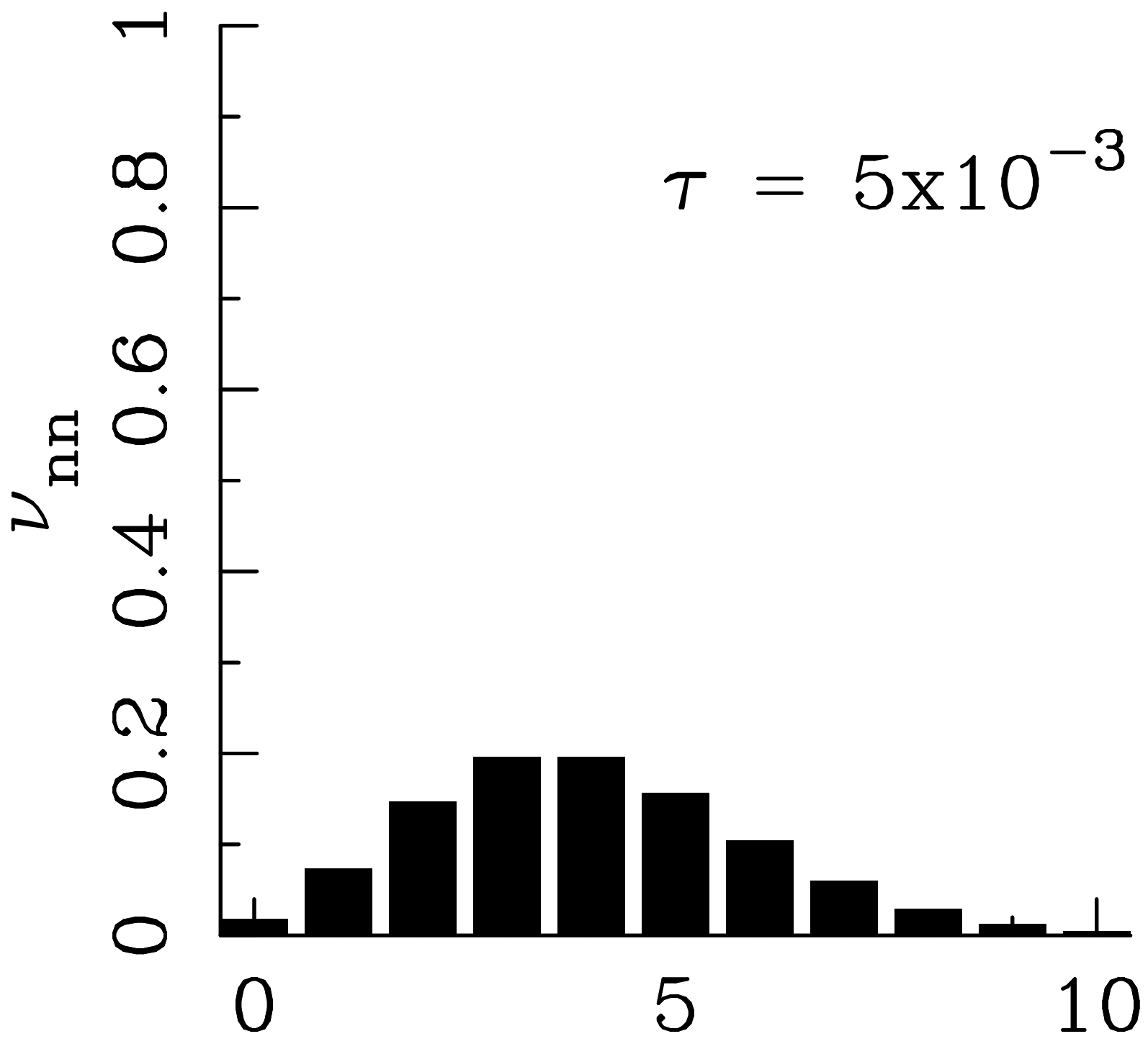

n 


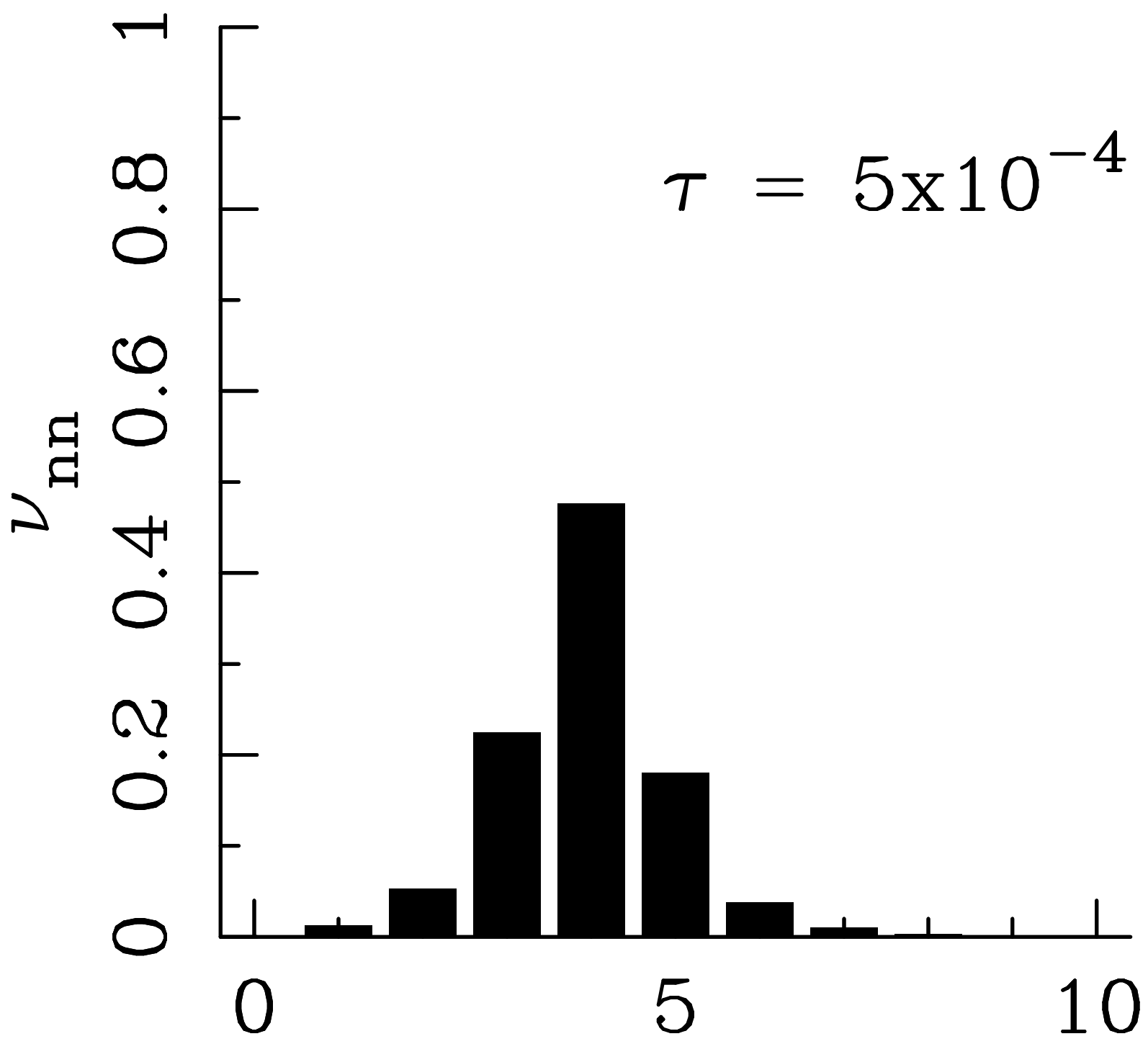

$\mathrm{n}$ 


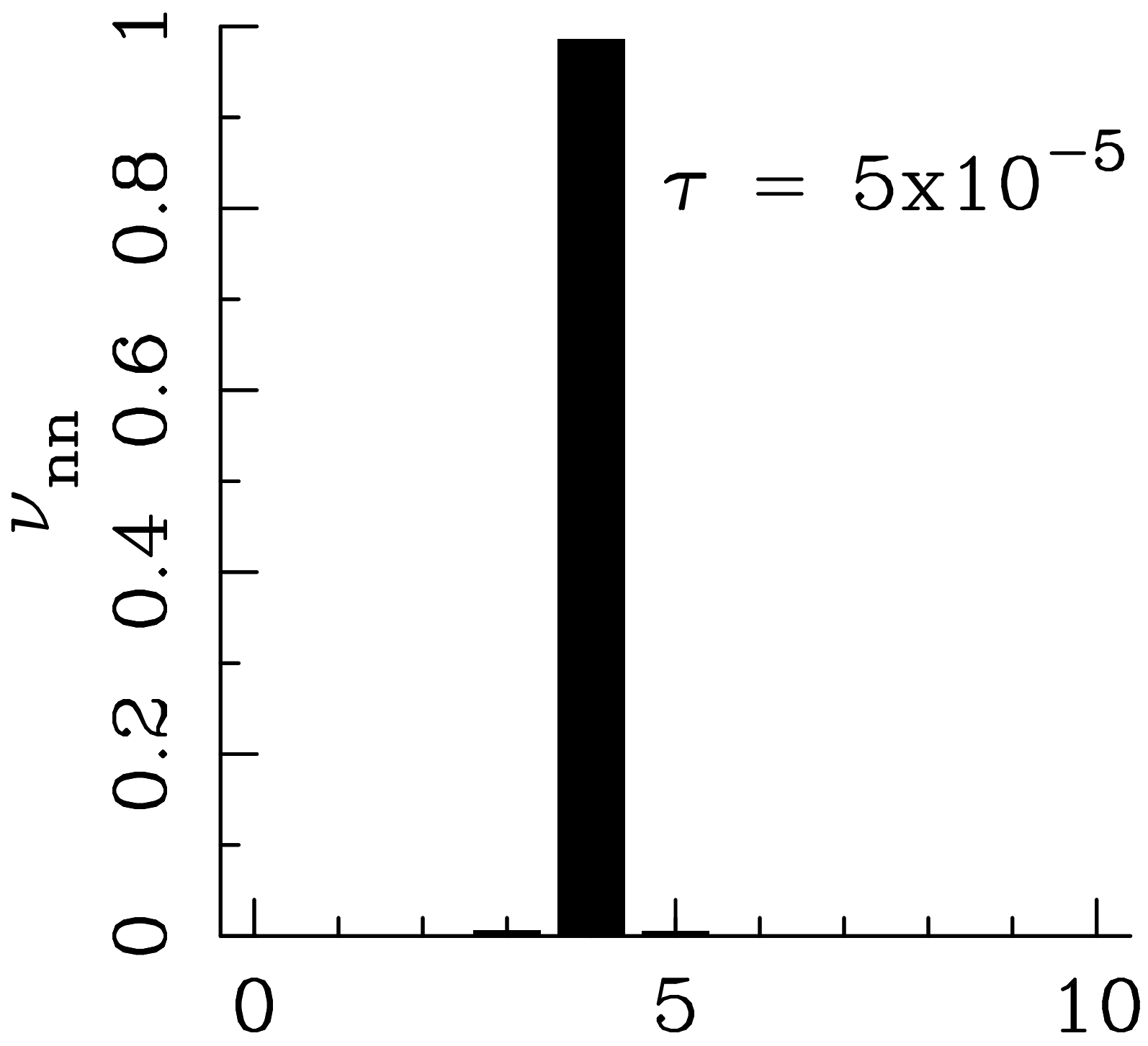

n 


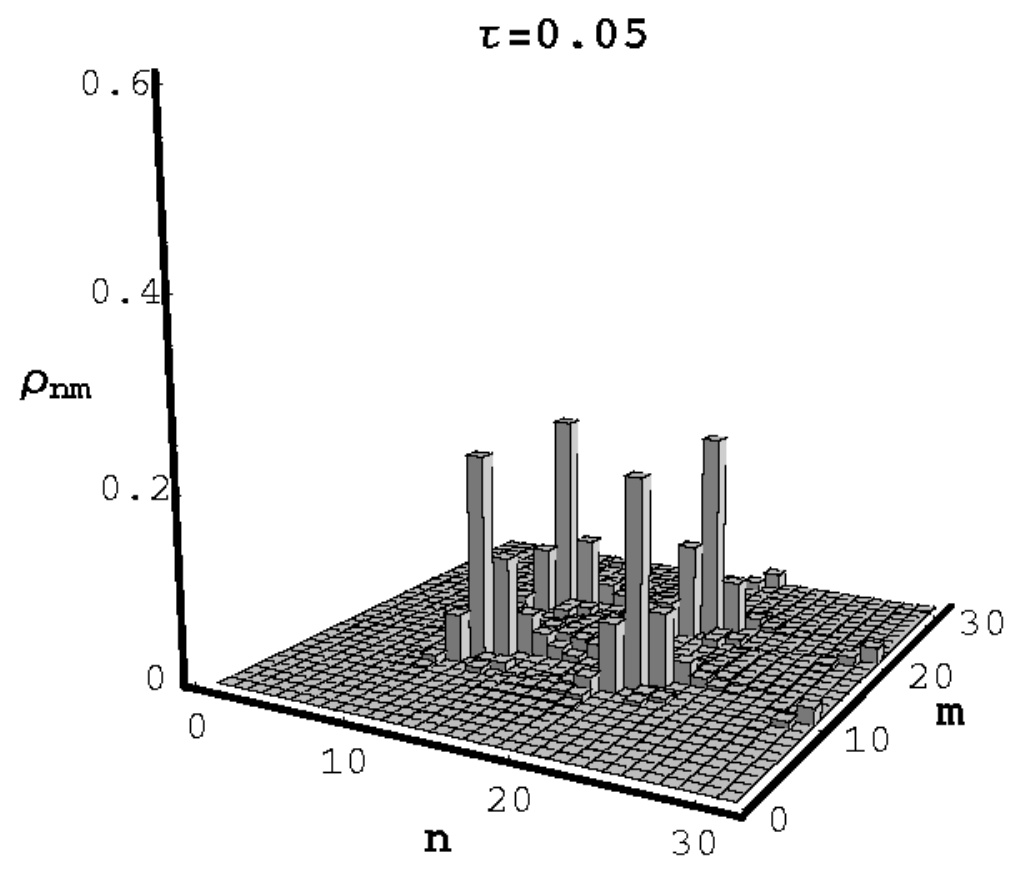




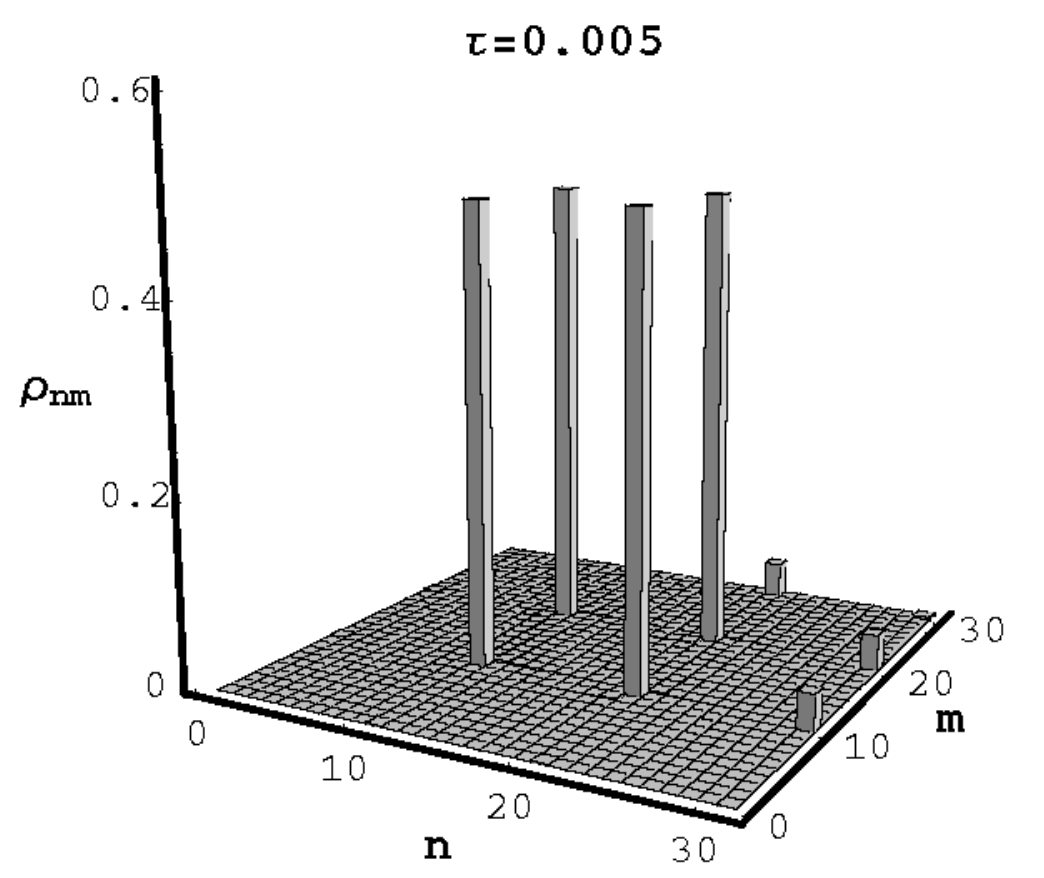




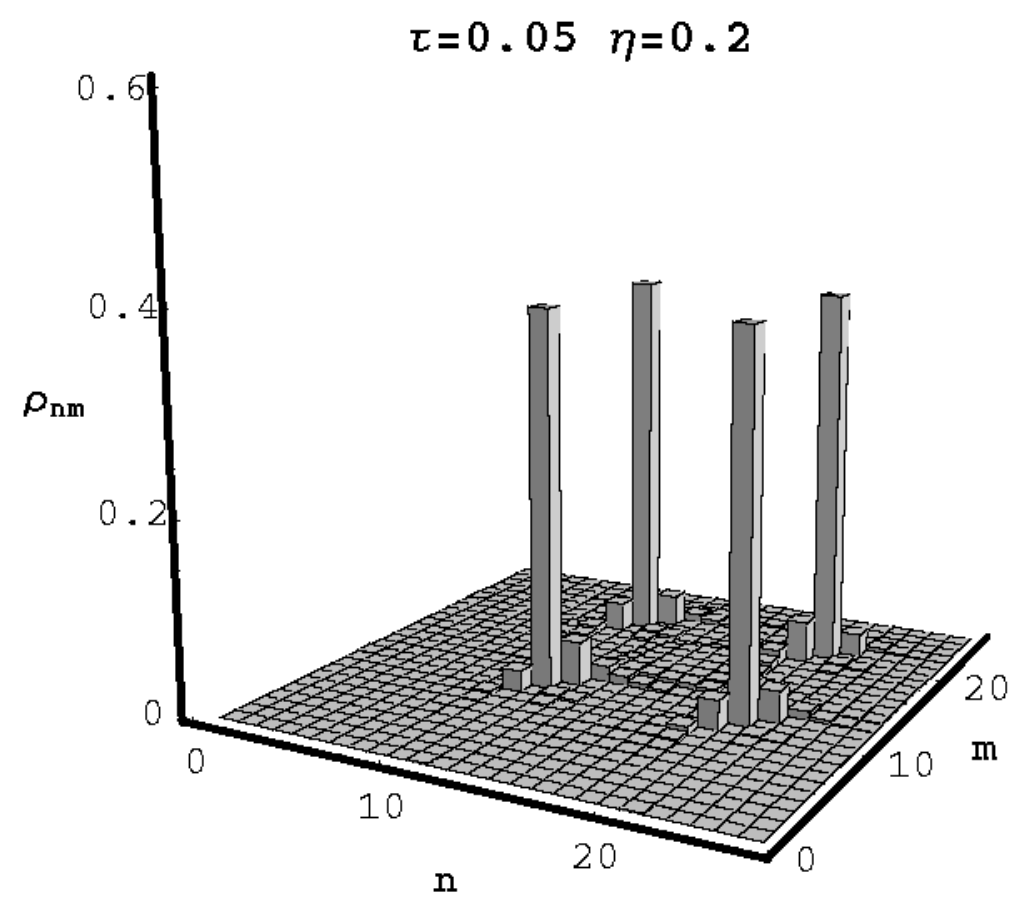




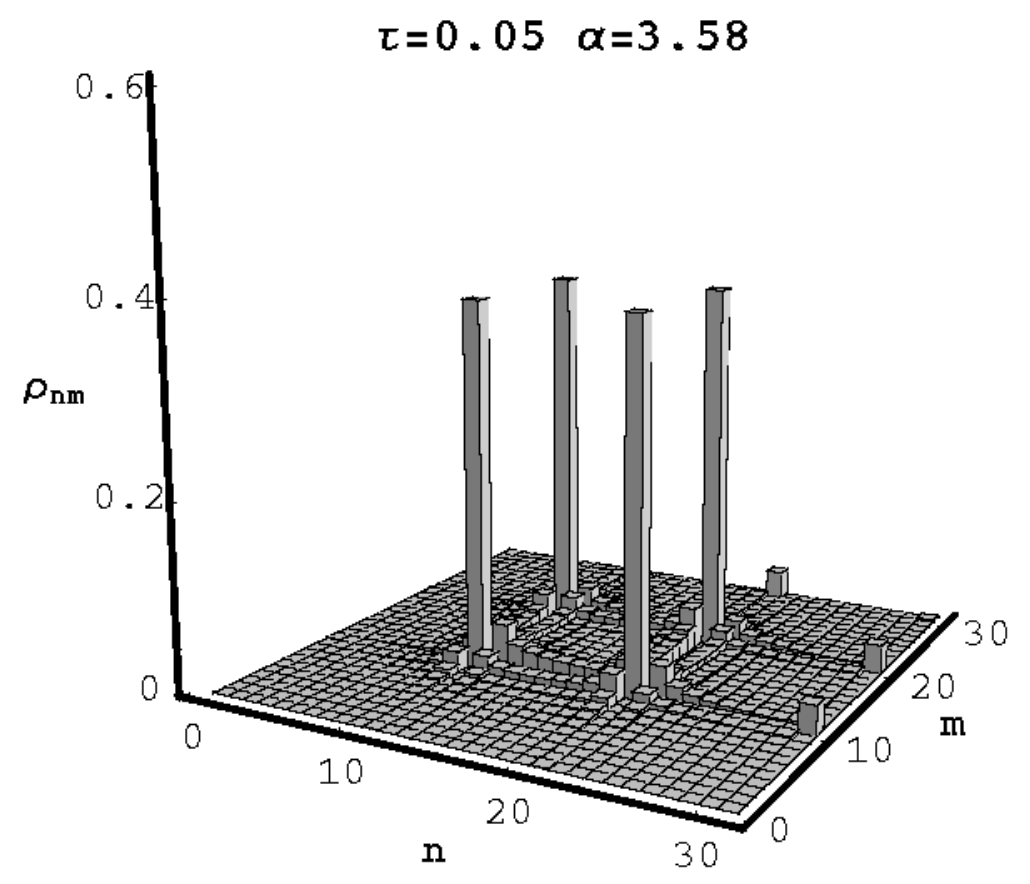

